\title{
A Cardiac Protection of Germinated Brown Rice During Cardiopulmonary Bypass Surgery and Simulated Myocardial Ischemia
}

\author{
Kanokwan Demeekul (D) \\ Pratch Sukumolanan (iD ${ }^{2}$ \\ Ratikorn Bootcha ${ }^{3}$ \\ Chattida Panprom ${ }^{3}$ \\ Soontaree Petchdee (iD) 4 \\ 'Graduate School, Program of Bio- \\ Veterinary Science, Kasetsart University, \\ Kamphaeng Saen, Nakorn Pathom, \\ Thailand; ${ }^{2}$ Veterinary Clinical Study \\ Program, Faculty of Veterinary Medicine, \\ Kasetsart University, Kamphaeng Saen, \\ Nakorn Pathom, Thailand; ${ }^{3}$ Kasetsart \\ University Veterinary Teaching Hospital, \\ Faculty of Veterinary Medicine, \\ Kamphaeng Saen, Nakorn Pathom, \\ Thailand; ${ }^{4}$ Department of Large Animal \\ and Wildlife Clinical Sciences, Faculty of \\ Veterinary Medicine, Kasetsart \\ University, KamphaengSaen Campus, \\ Kamphaeng Saen, Thailand
}

Correspondence: Soontaree Petchdee Department of Large Animal and Wildlife Clinical Sciences, Faculty of Veterinary Medicine, Kasetsart University,

KamphaengSaen Campus, Kamphaeng Saen, Nakorn Pathom, 73140, Thailand

Tel $+6634351901-3$

Fax +6634351405

Email fvetstr@ku.ac.th
Purpose: The potential cardio-protective property of germinated brown rice (GBR) has been revealed by ameliorating risk factors related to cardiovascular diseases. This study hypothesized that the combination of GBR and cardioplegic solution could protect the cardiomyocytes exposed to simulated ischemic reperfusion injury in vitro study and preserve cardiac function during cardiopulmonary bypass surgery in animal models.

Methods: Primary porcine cardiomyocytes were isolated and experimented cell viability against simulated ischemic reperfusion injury. In a cardiac surgical animal model, six pigs were randomly assigned to receive the two types of cardioplegic solution: i) St. Thomas cardioplegic solution $(20 \mathrm{cc} / \mathrm{kg})$; and ii) St. Thomas cardioplegic solution plus GBR (1 mg/ $\mathrm{kg}$ ). During open-heart surgery, the aorta was cross-clamped for 20 minutes, followed by reperfusion for 1 hour. Cardiopulmonary bypass parameters were recorded until the end of the procedure. Furthermore, hemodynamic parameters and arterial blood gas characteristics of animals among groups were monitored at different time points, including baseline before cardiopulmonary bypass (T1), during cardiopulmonary bypass (T2), during aortic clamp on (T3), and aortic clamp off (T4).

Results: Primarily, GBR cotreatment with cardioplegic solution essentially resulted in the improvement of cell viability in primary porcine cardiomyocytes against simulated ischemic reperfusion induction. The findings from cardiac surgery demonstrated that mean arterial pressure and heart rate are constantly stable in cardioplegic solution combined with the GBR group, while the trend of potassium and lactase concentration was decreased in the animals receiving GBR group. Consistently, all parameters from arterial blood gas showed better outcomes in animals receiving GBR; however, there were no statistically significant differences between groups, except hepatic enzymes.

Conclusion: Therefore, GBR might exert cardio-protective effects against ischemic reperfusion injury in the porcine cardiac surgery model due to anti-inflammatory response. These protective actions of GBR may explain the benefits gained from applying GBR products as a possible therapeutic supplement on cardiac diseases.

Keywords: cardioprotection, cardiopulmonary bypass, germinated brown rice, ischemic injury, primary porcine cardiomyocytes

\section{Introduction}

Cardiovascular diseases are among the most common causes of global death and continue rising in almost all countries. ${ }^{1}$ Ischemic heart disease is the major contributor to death in cardiovascular diseases. ${ }^{2}$ Besides, numerous risk factors such as hypertension, hyperglycemia, dyslipidemia, and sedentary lifestyle are well known 
as contributing causes of ischemic heart disease. ${ }^{3,4}$ Mainly, the standard therapeutic technique of cardiovascular diseases is a surgical treatment in humans. ${ }^{5}$ Usually, cardiopulmonary bypass (CPB) is extensively used during open-heart surgery. ${ }^{6}$ This technique encourages maintaining systemic perfusion and oxygenation during cardiac surgery under $\mathrm{CPB}{ }^{7}$ Myocardial protection is the critical principle, including reducing cell metabolism by hypothermia and cardiac arrest by cardioplegic solution. ${ }^{8}$

In practice, a cardioplegic solution's administration provides a motionless and bloodless operation field. ${ }^{9,10}$ Additionally, the cardioplegia solution exhibits the ability to reduce myocardial oxygen demand by creating electrical quiescence. Moreover, it has been established that cardioplegic solutions protect cardiomyocytes against ischemic reperfusion injury during CPB surgery. ${ }^{11,12}$

To date, exploring the health benefits of functional foods has attracted increasing attention, especially phytochemical substrates. ${ }^{13,14}$ Germinated brown rice (GBR) is a biotransformation product of brown rice and an excellent source of phytochemical agents. ${ }^{15}$ In recent years, many researchers have reported the protective effects of germinated brown rice (GBR), such as antihypertension, anti-atherosclerotic, anti-oxidant, antiobesity, antidepressant, anti-inflammatory, antidiabetic, and hypolipidemic. ${ }^{16-20}$ According to several studies, it has been established that GBR exerts its health benefits due to bioactive compounds such as phenolic compounds, total flavonoid, $\gamma$-Aminobutyric acid (GABA), and phytic acid. ${ }^{13,15,21}$ Furthermore, our previous studies have initially demonstrated potential cardioprotective properties of GBR extract both in myocardial ischemicreperfusion injury and chronic myocardial infarction. The possible mechanism is the activation of $\mathrm{p} 38$ MAPK and downstream of proapoptotic Bax and Bcl-2, and caspase- 3 protein expression. ${ }^{21,22}$ Recently, a study in $\mathrm{H} 9 \mathrm{c} 2$ cardiomyocytes subjected to simulated ischemic reperfusion injury has documented a protective property of GBR via ameliorated mitochondria dysfunction by maintaining mitochondrial membrane potential and preserving mitochondrial respiration. ${ }^{23}$ Accumulating indicated that GBR might preserve the cardiomyocytes' function from ischemic reperfusion injury in an experimental model. However, the effects of GBR on cardiac protection during cardiac surgery under $\mathrm{CPB}$ have not been studied.

Therefore, this study investigates whether GBR and cardioplegic solution's cotreatment protects cardiomyocyte injury during open-heart surgery under $\mathrm{CPB}$ in the porcine experimental model.

\section{Materials and Methods}

\section{Animals}

Animal experiments were conducted at Kasetsart University Veterinary Teaching Hospital, the Faculty of Veterinary Medicine, Kasetsart University, Kamphaeng Saen campus. The animal ethics was approved by Kasetsart University Institutional Animal Care and Use Committee, Kasetsart University, Bangkok, Thailand, used committee "IACUC" approval number ACKU62VET-059, and followed the guidelines for the use of animals National Research Council of Thailand policy and procedures for animal care. All surgery procedures were performed under anesthesia, minimizing pain, suffering, and distress of the animal. Nursery pigs $(2-10 \mathrm{~kg}$ ) were provided by the Faculty of Veterinary Medicine, Kasetsart University, Kamphaeng Saen campus. Heart isolation was used to study the cell viability of primary porcine cardiomyocytes in GBR co-treatment with cardioplegic solution in simulated ischemic-reperfusion conditions. On the other hand, six adult pigs (30$45 \mathrm{~kg}$ ) were obtained, and cardiopulmonary bypass surgery was performed for open heart surgery in an animal model.

\section{Isolation of Primary Porcine Cardiomyocyte}

The heart was routinely dissected with an aseptic technique. Isolation of cardiomyocytes protocol was modified from a previous study. ${ }^{24,25}$ Briefly, the right atrium chamber was immediately identified and isolated into a sterile glass Petri dish. After that, the isolated right atrium was gently washed with PBS mixed with $0.5 \mathrm{~mL}$ of $10 \%$ marbofloxacin three times. This isolated right atrium was chopped into small pieces and digested with $0.4 \%$ type II collagenase and $0.01 \%$ DNAse. Finally, pellets of the right atrium were plated to a T25 culture flask in DMEM culture medium containing $10 \%$ FBS, amphotericin, penicillin G, and streptomycin under $\mathrm{pH} 7.4,37^{\circ} \mathrm{C}$ in humidified air containing $5 \% \mathrm{CO}_{2}$. The $70 \%$ confluence of primary porcine cardiomyocytes cells growth were used for all experiments in passage number $1-2$. The culture medium was changed with warm fresh medium every 3 days. Cells were cultured under either GBR pretreatment, cardioplegic solution, or $\mathrm{sI} / \mathrm{R}$ induction. 


\section{Simulation of Ischemic Reperfusion Injury in Primary Porcine Cardiomyocyte}

In this study, ischemic simulation was conducted as described in previous research. ${ }^{26}$ In an ex vivo study, we provided sodium dithionite to induce the ischemic condition. Briefly, primary porcine cardiomyocytes were cultured in DMEM with 10\% fetal bovine serum (FBS) for all experiments. These primary cells were incubated in $200 \mu \mathrm{l}$ of ischemic buffer $(137 \mathrm{mM} \mathrm{NaCl}, 3.8 \mathrm{mM} \mathrm{KCl}$, $0.49 \mathrm{mM} \mathrm{MgCl}_{2}, 1.8 \mathrm{mM} \mathrm{CaCl}_{2}$, and $4.0 \mathrm{mM}$ HEPES), followed by $20 \mathrm{mM}$ of 2-deoxyglucose, $30 \%$ sodium lactate, and $1 \mathrm{mM}$ sodium dithionite at $\mathrm{pH} 6.3$, to induce $\mathrm{sI} / \mathrm{R}$ in a 96-well plate. The effective ischemic induction period was optimized in different time points ranging from 10 to 120 minutes. After ischemic induction, the ischemic buffer was replaced and the cells were incubated with cultured medium. The appropriated time for $\mathrm{sI} / \mathrm{R}$ induction was performed for all ex vivo studies.

\section{DNA Extraction and Polymerase Chain Reaction (PCR)}

To verify the primary porcine cardiomyocyte, cardiac myosin binding protein $\mathrm{C} 3$ gene (cMYBPC3) was preliminary performed by polymerase chain reaction (PCR) method, as described previously. ${ }^{27}$ According to the manufacturer's instructions, DNA extraction was accessed with a DNeasy blood and tissue kit (Qiagen, Germany). Amplification of genomic DNA was performed using the following primer set: forward primer 5' AGCCTTCAGCAAGAAGCCA3' and reverse primer 5' CAAACTT GACCTTGGAGGAGC3'. The estimated size of the PCR product is 242 base pairs. The PCR condition was carried out with $2.5 \mu \mathrm{L}$ of DNA template in a total volume of $25 \mu \mathrm{L}$, also containing $12.5 \mu \mathrm{L}$ of HotStarTaq Master Mix (Qiagen, Germany), $0.5 \mu \mathrm{L}$ of forward primer $(0.2 \mu \mathrm{M}), 0.5 \mu \mathrm{L}$ of reverse primer $(0.2 \mu \mathrm{M})$, and $9 \mu \mathrm{L}$ of RNase-Free water (Qiagen, Germany). PCR for cMYBPC3 gene $\left(95^{\circ} \mathrm{C} 15\right.$ minutes, $95^{\circ} \mathrm{C} 30 \mathrm{sec}-$ onds, $58^{\circ} \mathrm{C} 30$ seconds; 35 cycles, $72^{\circ} \mathrm{C} 15$ minutes) was performed on the sample. The PCR products were electrophoresed on $1.5 \%$ agarose gel at 135 volts for 20 minutes and stained by ethidium bromide to visualize DNA bands with a Gel Doc EZ Gel Documentation System (Biorad, USA).

\section{Germinated Brown Jasmine Rice (GBR) Extract}

GBR extract was provided by the cardiac research laboratory unit, Faculty of Veterinary Medicine, Kasetsart University,
Kamphaeng Saen campus. The preparation of GBR extract was obtained as depicted in our previous work. ${ }^{21}$ In brief, Brown jasmine rice (Oryza sativa L.) was washed, and $100 \mathrm{~g}$ of cleaned grains were soaked in water at $35^{\circ} \mathrm{C}$ for 12 hours. After that the seeds were placed on moist filter papers and covered for 24 hours of germination. Then, the germinated seeds were finely ground, prior to preparing the germinated brown rice extract powder. The samples were stored at $-20^{\circ} \mathrm{C}$ until used. Finally, the water-soluble GBR extraction was filtered using a sterilized acrylic membrane syringe filter with $0.2 \mu \mathrm{m}$ pore size.

\section{Light Microscopy and Acquisition of Pictures}

Digital images were received using a phase-contrast microscope for cell culture (10X, Nikon Eclipse TS100, USA) with a digital camera modulated by NIS software. Cells were seeded in a 96-well plate at a density of $4 \times 10^{4}$ cells in 200 $\mu \mathrm{L}$ cultured medium/well and incubated according to the different concentrations of cardioplegic solution and experimental protocols. Representative photographs were taken of three different parts of incubated cell culture dishes.

\section{Determination of Cell Viability}

Due to cell viability measurement, tetrazolium bromide (MTT) assay was performed based on mitochondria dehydrogenase activity. Primary porcine cardiomyocytes were seeded at a density of $4 \times 10^{4}$ cells/well in a 96-well plate until $80 \%$ confluence was reached. The suitable time for simulated ischemic reperfusion injury was done by incubating cells with $\mathrm{sI} / \mathrm{R}$ at several time points and cell viability was accessed by MTT assay. In addition, the cells were incubated for 24 hours either in the $5 \mathrm{ng} / \mathrm{mL}$ of GBR extract or $1 \%$ St. Thomas cardioplegic solution or both before being subjected to sI/R. After that the culture media was discarded, $0.5 \mathrm{mg} / \mathrm{mL}$ MTT reagent was added, and incubated for 2 hours at $37^{\circ} \mathrm{C}$. At the end of the incubation period, the excess MTT reagent was removed and DMSO was added to solubilize the formazan dye. The optical density (OD) was determined by a spectrophotometer at $\lambda 595 \mathrm{~nm}$ using DMSO as a blank. The relative percentage of cell viability was compared against the control group.

\section{Cardiopulmonary Bypass (CPB) Surgery in a Porcine Animal Model}

The surgical procedure to open the heart was performed according to a well-accepted technique with modifications. ${ }^{28}$ 
Briefly, pigs were anesthetized with propofol $(0.2 \mathrm{mg} / \mathrm{kg})$ and underwent tracheal intubation for subsequent mechanical ventilation. The tidal volume and the minute volume were adjusted to produce an arterial carbon dioxide tension of $35-45 \mathrm{~mm} \mathrm{Hg}$. Anesthesia and paralysis were maintained by inspiration of isoflurane (1-5\%) and intermittent boluses of fentanyl $(2 \mu \mathrm{g} /$ $\mathrm{kg})$. An arterial line was inserted into the left femoral artery and a central venous line was inserted into the right atrium. Electrocardiogram, arterial blood pressure, oxygen saturation from pulse oximetry $\left(\mathrm{SpO}_{2}\right)$, heart rate, and rectal temperature were monitored throughout the procedure.

The operations with cardiopulmonary bypass (CPB) included a roller pump, arterial cannulation, venous cannulation, and mild systemic hypothermia $\left(32-36^{\circ} \mathrm{C}\right)$ (Figure 1). The CPB circuit was filled with a priming solution consisting of $50 \mathrm{~mL}$ of sodium bicarbonate, $50 \mathrm{~mL}$ of gelofusine, $20 \%$ mannitol $(5 \mathrm{~mL} / \mathrm{kg})$, and $800 \mathrm{~mL}$ acetate Ringer's solution. Heparin (300 IU/kg) was administered to maintain the activated clotting time over 480 seconds. ${ }^{29} \mathrm{CPB}$ was initiated at a flow rate of $100-150 \mathrm{~mL} / \mathrm{kg} / \mathrm{min}$ and adjusted to produce a minimal mean arterial pressure of $50 \mathrm{mmHg}$. Pigs in the experimental groups were cooled to $32^{\circ} \mathrm{C}$. Cardiac arrest was achieved by ascending aorta crossclamping for 20 minutes and adding either St. Thomas cardioplegia solution $(20 \mathrm{cc} / \mathrm{kg})$ or combined with GBR $(1 \mathrm{mg} / \mathrm{kg})$ into the ascending aorta for cardiomyocytes protection during the 20 minutes interval of cardiac arrest. Hemodynamic parameters and arterial blood gas were monitored dividing into four phases including $\mathrm{T} 1$; before $\mathrm{CPB}$, T2; during CPB, T3; aortic clamp-on, T4; aortic clamp off. When the animals were cooled to the rectal temperature of $32^{\circ} \mathrm{C}$, the arterial flow rate decreased to $50 \mathrm{~mL} / \mathrm{kg} / \mathrm{min}$. After releasing the cross clamp, the animals were rewarmed to a core temperature of $36-37^{\circ} \mathrm{C}$ and weaned from the CPB circuit. The animals were euthanized 2 hours after the clamp was removed.

\section{Statistical Analysis}

All experiments were performed at least three independent experiments. All data are presented as means \pm standard deviation (SD). Comparisons among groups were assessed for significance using one-way analysis of variance (ANOVA) followed by Tukey Post hoc test. Some parameters, which were compared between two groups or different time points, were measured for significance using a two-way analysis of variance (ANOVA). GraphPad Prism 8 software was used for all statistical analyses and $p<0.05$ was taken to indicate a statistically significant difference.

\section{Results \\ DNA Amplification of cMYBPC3 Gene}

cMYBPC 3 is a cardiac isoform of myosin binding protein C. ${ }^{30}$ This protein plays a role in the regulation of cardiac contraction. The mutation of $\mathrm{cMYBPC} 3$ had reported in the association with familial hypertrophic cardiomyopathy and was found to be dephosphorylated in I/R injury. ${ }^{31}$ In this study, we performed DNA amplification of cMYBPC3 gene by PCR method as described before. In particular, PCR condition has successfully amplified the fragments of cMYBPC3 in primary porcine cardiomyocyte isolated from the right atrium (RA). The gel electrophoresis detection exhibited the target band at $242 \mathrm{bp}$ as shown in Figure 2. This result suggested that the cMYBPC 3 gene was detected in primary porcine cardiomyocytes.

\section{Optimization of Time for Simulated Ischemia/Reperfusion (sl/R) Injury in Primary Porcine Cardiomyocytes}

Primary porcine cardiomyocytes were subjected to different time points of simulated ischemia ( $0-120$ minutes), followed by cell viability measurement. The results demonstrated that simulated ischemia decreased cell viability in a timedependent manner (Figure 3). The $\mathrm{sI} / \mathrm{R}$ at times varying from 10 minutes to 30 minutes did not affect the cell viability of primary porcine cardiomyocytes than control. The sI/R periods from 40 minutes to 120 minutes significantly reduced the percentage of cell viability, respectively. In this study, we preferred the condition of $s I / R$ at 40 minutes as a $s I / R$ protocol for examining the sI/R injury-induced cell death.

\section{Optimization of Cardioplegic Concentration in Primary Porcine Cardiomyocytes}

To further evaluate the optimized concentration of a cardioplegic solution, primary porcine cardiomyocytes were incubated with various percentages of cardioplegic solution, consisting of $1 \%$ to $50 \%$ for 24 hours. At the end of incubation times, cell morphology and the percentage of cell viability were acquired. The microscopic results were demonstrated in Figure 4A while the percentage of cell viability was represented in Figure 4B. The primary porcine cardiomyocytes in control condition showed a spheroidal shape, while $1 \%$ of cardioplegic condition did not change the morphology of cardiomyocytes. These results were constantly similar to the percentage of cell 


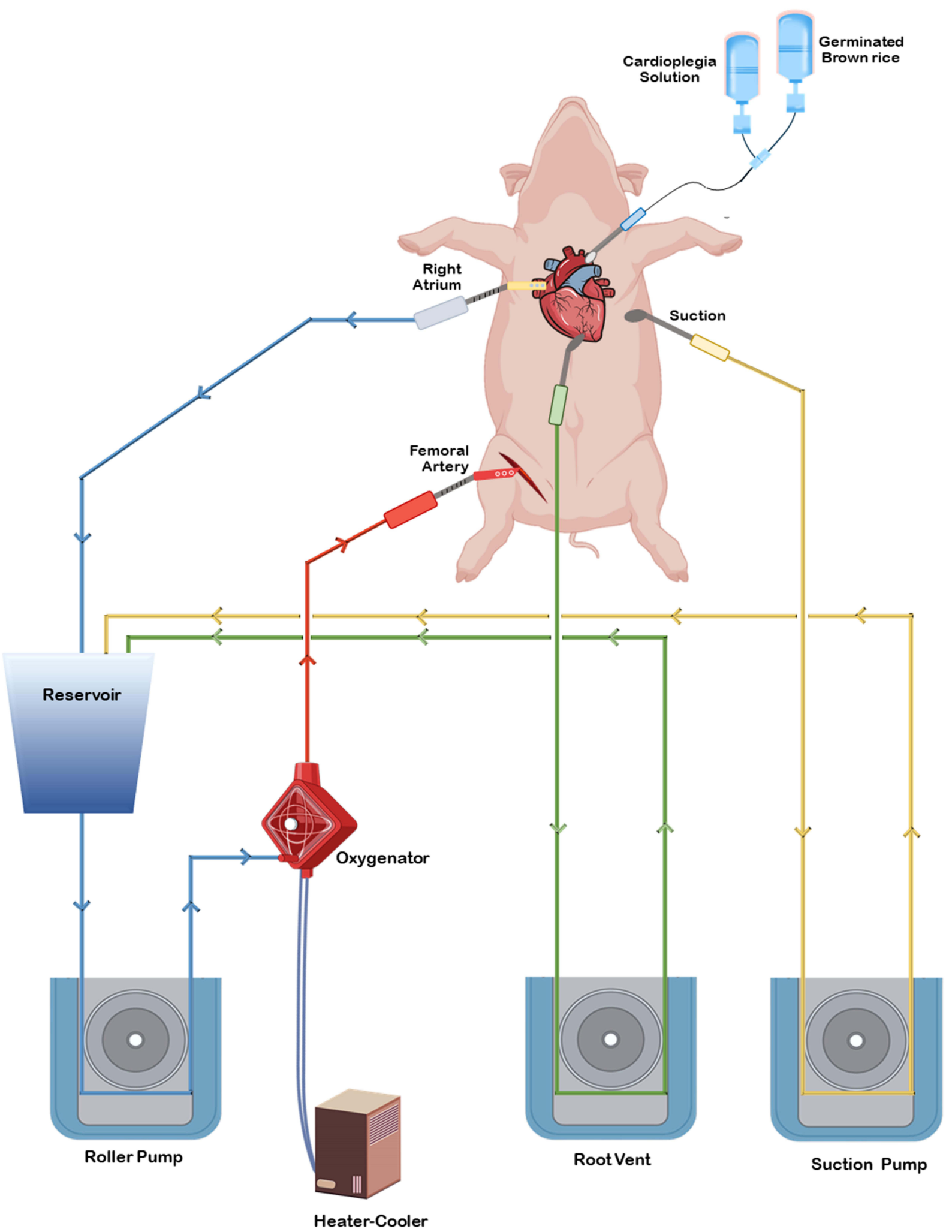

Figure I Diagrammatic representation of cardiopulmonary bypass surgery in a porcine experimental model. The open-heart surgery procedure was performed on the median sternotomy. An arterial cannula was placed in a femoral artery, and a venous cannula was inserted into the right atrium. Both cannulas were connected to the CPB circuit. Venous blood was drained from the right atrium cannula to the blood reservoir and delivered to the oxygenator, the artificial lung, by the CPB pump. The blood was then returned to animal circulation via the femoral artery. Spill-blood was returned to the blood reservoir through a suction line. Blood in the heart chambers was drained by vent line. Cardioplegic solution and GBR solution were supplied to protect the myocardium and stop the heart beating during surgery. 


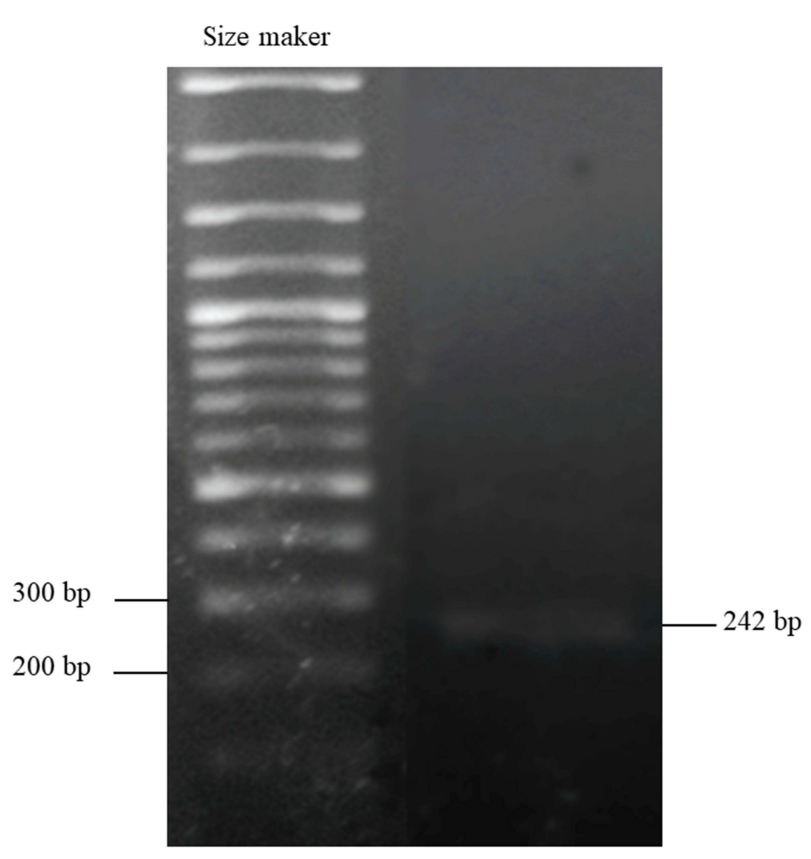

Figure 2 DNA amplification by PCR examined in primary porcine cardiomyocyte cell isolated from the right atrium (RA). Fragment of the expected size for MYBPC3 gene (242 base pairs) was amplified successfully in the sample.

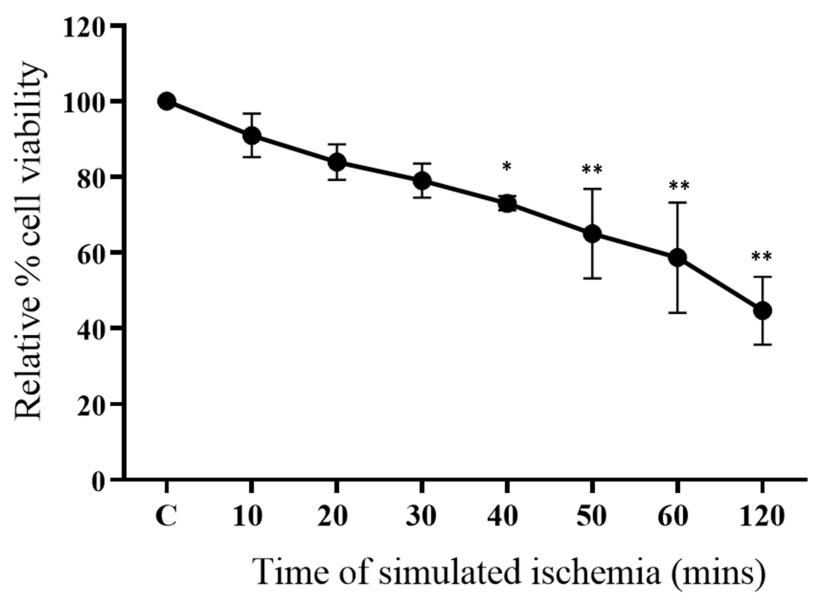

Figure 3 Optimization of time for simulated ischemia/reperfusion (sl/R) injury in primary porcine cardiomyocytes. The cells were incubated with different time points varying from 10 to 120 minutes. The percentage of cell viability was measured by MTT assay $(n=4)$. Each bar graph represents the mean \pm SD. One-way ANOVA analyzed data with Tukey's Multiple Comparison Test, $*_{p}<0.01$, $* * p<0.000$ I compared to control.

viability (Figure 4B). Interestingly, 5\% and 10\% cardioplegic condition initially led to widespread and flat morphology of primary porcine cardiomyocytes. Damage with $25 \%$ and $50 \%$ of cardioplegia solutions sharply resulted in membrane destruction and development of apoptotic bodies (Figure 4A). Consistently, the percentage of cell viability remarkedly decreased in various concentrations of cardioplegic solutions, varying from $5 \%$ to $50 \%$, respectively (Figure 4B). Therefore, we provided a cardioplegic solution concentration at $1 \%$ for downstream further experiments in this study.

\section{Effect of GBR Co-Treatment with Cardioplegic Solution in Primary Porcine Cardiomyocytes}

The cardioprotective effect of GBR has been revealed in a previous study. ${ }^{21,22}$ However, the effect of GBR treatment combined with cardioplegic solution has not been explored yet. To investigate whether the combination of GBR and cardioplegic solution protected against sI/R injury, cell viability of primary porcine cardiomyocytes cultured in experimental conditions for 72 hours were determined by MTT assay. As shown in Figure 5A and Figure 5B, a marked reduction of cell viability was detected in primary porcine cardiomyocytes subjected to sI/R condition compared with the control condition (100\% \pm 0 vs $63.34 \% \pm 6.22$ ). This result agreed with the appearance of cell morphology results from light microscopy (Figure 5A). Pretreatment of either GBR or cardioplegic solution significantly greater brought the percentage of cell viability back to a similar level of primary porcine cardiomyocytes cultured in the control group $(100 \% \pm 0$ vs $84.35 \% \pm 3.48,100 \% \pm 0$ vs $86.86 \% \pm 4.00$, respectively). Consequently, cells in cotreatment with GBR and cardioplegic solution subjected to $\mathrm{sI} / \mathrm{R}$ exhibited an effective increase in cell viability compared to the control condition $(100 \% \pm 0$ vs $84.44 \% \pm 4.87$ ) (Figure $5 \mathrm{~B}$ ). This result suggested that either GBR combined with cardioplegic solution or those alone in these treatments exerts a protective effect against sI/R induced primary porcine cardiomyocytes injury in a similar fashion.

\section{Effect of GBR Treatment on Cardiopulmonary Bypass Surgery}

The cardioprotective effect of GBR has been unveiled on primary porcine cardiomyocytes subjected to sI/R. To gain more insight and further confirm the cardioprotective effect of GBR, cardiopulmonary bypass surgery was performed in an experimental animal model. Six pigs were randomly divided into two groups. The control group (Con, $\mathrm{n}=3$ ) received a St. Thomas cardioplegic solution, while the GBR treatment group (GBR, $n=3$ ) received both GBR and St. Thomas cardioplegic solution. 
A

Control

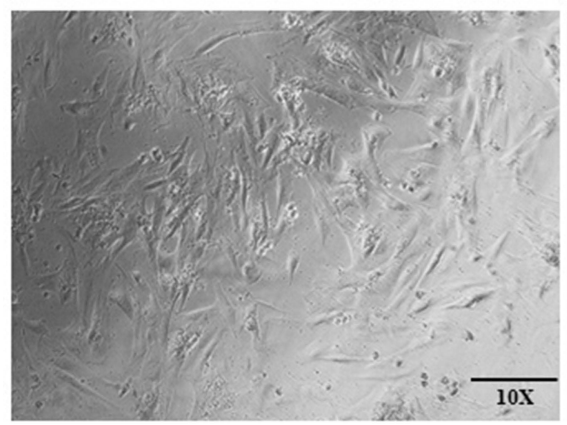

$10 \%$ CPG

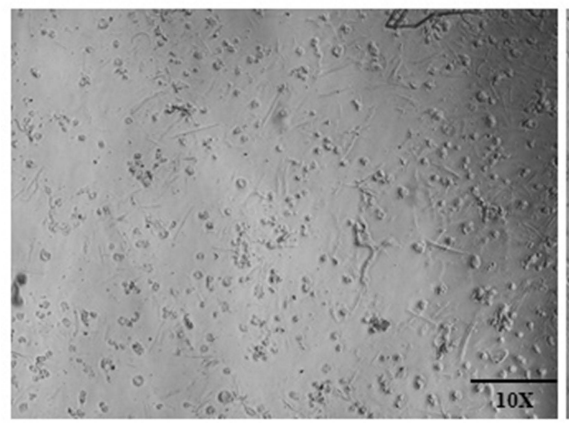

$1 \%$ CPG

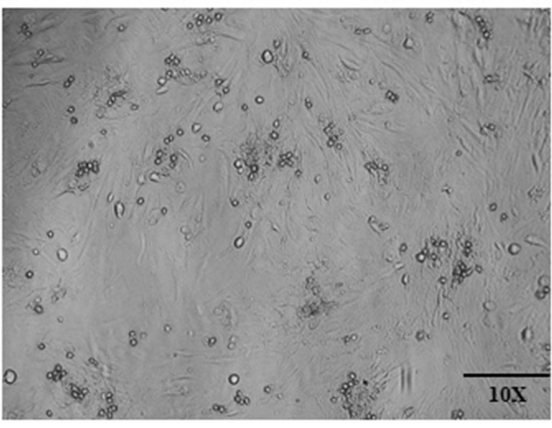

$25 \%$ CPG

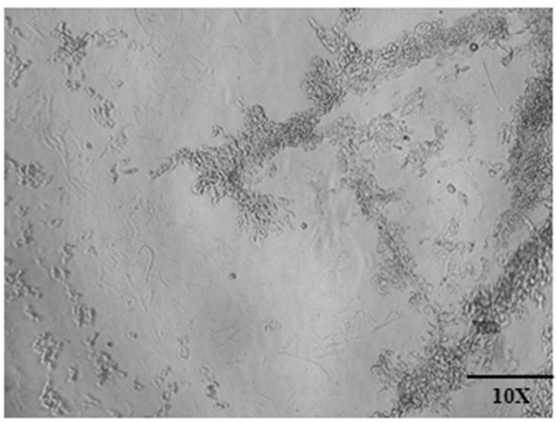

$5 \%$ CPG

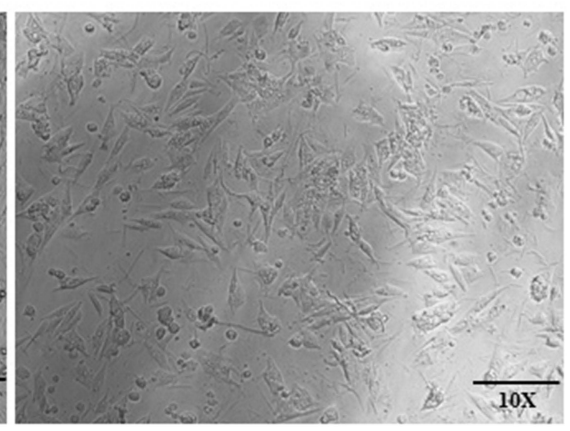

$50 \%$ CPG

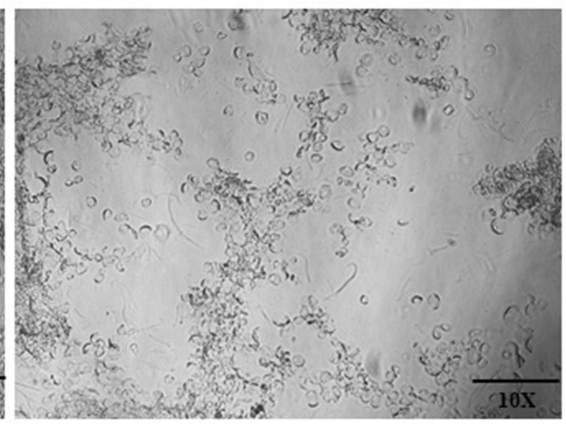

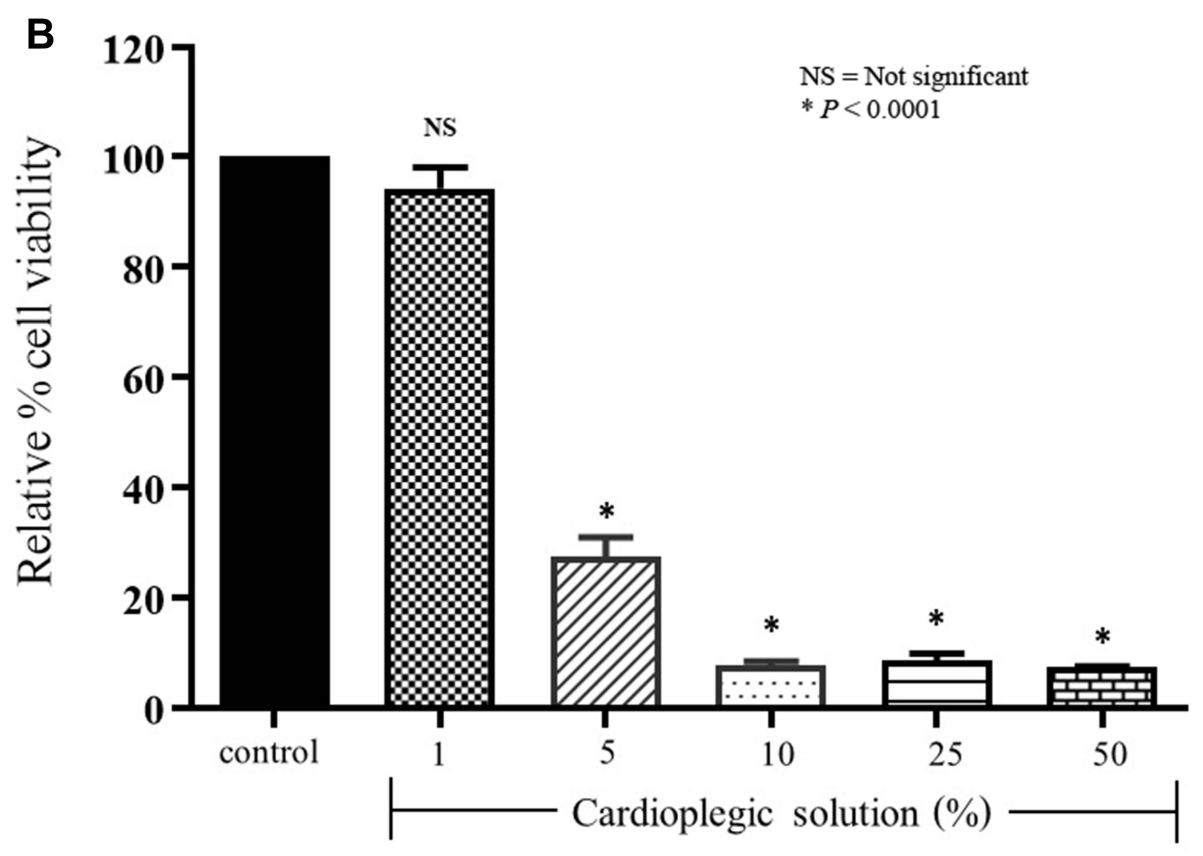

Figure 4 Optimization of cardioplegic solution concentration in primary porcine cardiomyocytes. The cells were cultured in various cardioplegic solution concentrations, consisting of $\mathrm{I}-50 \%$ for 24 hours. (A) The change of cell morphology was captured under microscopy (I0X). (B) The percentage of cell viability was examined by MTT assay $(n=3)$. Each bar graph represents the mean \pm SD. One-way ANOVA analyzed data with Tukey's Multiple Comparison Test, NS = not significant, * $p<0.000 \mathrm{I}$ compared to control.

\section{Effect of GBR Treatment on Clinical Characteristics of the Animal Model}

The clinical characteristics of animals undergoing cardiopulmonary bypass (CPB) surgery are represented in Table 1. The result showed increased body weight, heart rate, total cardioplegia volume, and dopamine dose in the GBR group compared to those who received GBR. On the other hand, some CPB parameters, including cooling time, rewarming time, and $\mathrm{CPB}$ time, demonstrated an assistive trend in GBR group animals. 


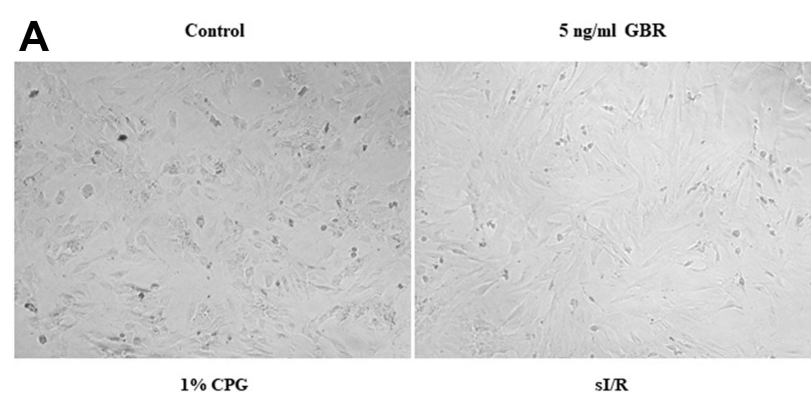

$1 \% \mathrm{CPG}$

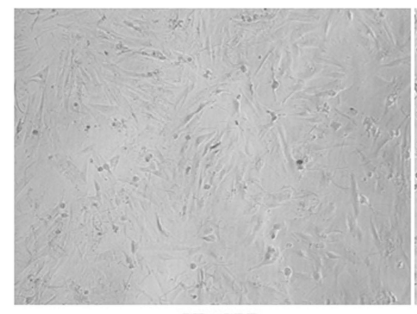

sI/R+GBR

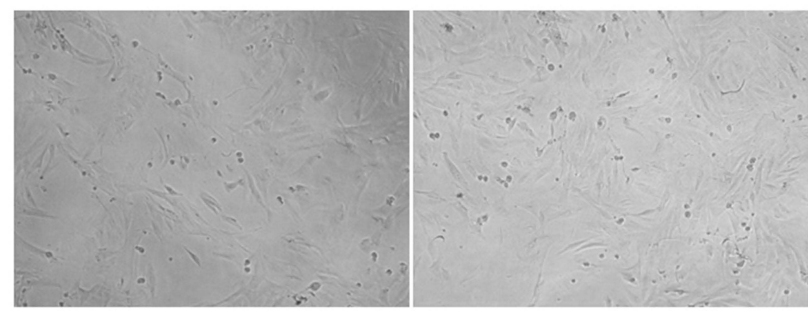

sI/R+GBR+CPG

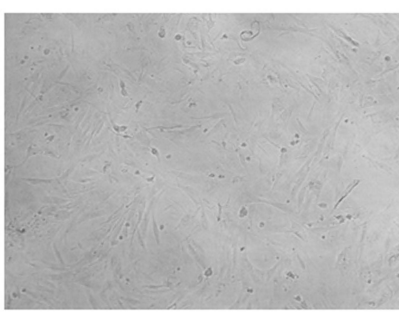

SI/R

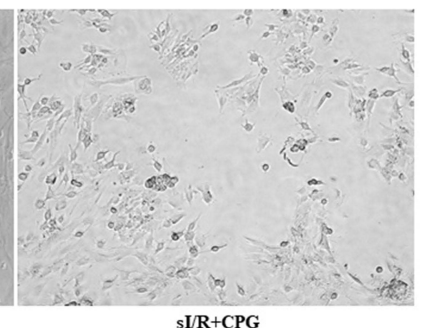

$s \mathbf{I}+\mathbf{C P G}$
B

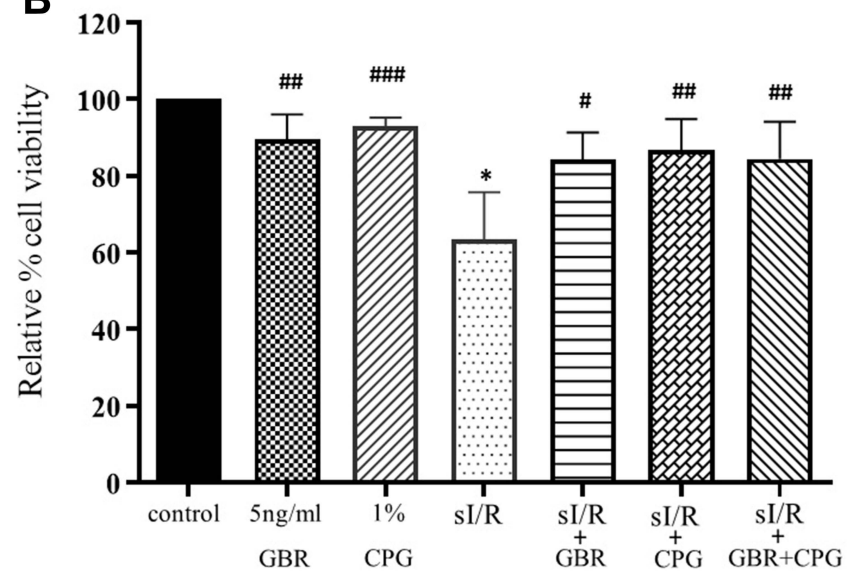

Figure 5 Effect of GBR cotreatment with cardioplegic solution in primary porcine cardiomyocytes subjected to sl/R injury. The cells were pretreated with GBR and cardioplegic solution for 24 hours. (A) The change of cell morphology was captured under microscopy (I0X). (B) The percentage of cell viability was accessed by MTT assay $(n=4)$. Each bar graph represents the mean \pm SD. One-way ANOVA analyzed data with Tukey's Multiple Comparison Test, * $p<0.000 \mathrm{I}$ compared to control; \#p<0.05, $\#$ \#P<0.01, \#\#<0.00I compared to sl/R.

However, there was no significant difference in all parameters.

\section{Effect of GBR Treatment on Hemodynamic Data of Animal Model}

Hemodynamic parameters and arterial blood gas were monitored until the end of the experimental procedure. There are four phases of time points, including $\mathrm{T} 1$; before $\mathrm{CPB}, \mathrm{T} 2$; during $\mathrm{CPB}, \mathrm{T} 3$; aortic clamp-on, T4; aortic clamp off. Heart rate and mean arterial pressure showed a fluctuated pattern several times in the control and GBR group (Figure 6A and B). Consistent with Table 2, parameters of $\mathrm{pH}, \mathrm{Pco}_{2}, \mathrm{Po}_{2}$, and oxygen saturation were not statistically significant differences between the two groups at different time points. Moreover, animals in the GBR group represented a reduction of sodium, potassium, and lactase concentration. These results suggested that animals receiving GBR plus cardioplegic solution could exert a more significant potential cardioprotective effect.

\section{Effect of GBR Treatment on Blood Chemistry Profile of the Animal Model}

The blood chemistry profile was observed in the control group and GBR group, as shown in Table 3. This result represented a higher value of white blood cell count, platelets, and BUN, while the percentage of hematocrit, creatinine, and plasma protein was decreased in the GBR group compared to the control group. However, there were 
Table I Clinical Characteristics of Animals

\begin{tabular}{|l|c|c|c|}
\hline Characteristics & CON & GBR & p-value \\
\hline Body weight (kg) & $35.33 \pm 5.33$ & $40.27 \pm 4.63$ & 0.85 \\
Heart rate (BPM):TI & $91.33 \pm 10.73$ & $123.67 \pm 23.25$ & 0.35 \\
Cooling time (min) & $35.00 \pm 2.89$ & $30 \pm 10.00$ & 0.15 \\
Rewarming time (min) & $31.67 \pm 7.26$ & $15 \pm 2.89$ & 0.27 \\
Cross-clamping time (min) & $20.00 \pm 0.00$ & $20.00 \pm 0.00$ & - \\
CPB time (min) & $66.67 \pm 6.67$ & $60 \pm 5.77$ & 0.85 \\
Total cardioplegia volume & $233.33 \pm 16.67$ & $250 \pm 28.87$ & 0.50 \\
(mL/kg) & & & \\
Dopamine dose ( $\mu \mathrm{g} / \mathrm{kg})$ & $0.33 \pm 0.33$ & $2 \pm 1.00$ & 0.20 \\
\hline
\end{tabular}

Abbreviations: CON, control group; GBR, Adding GBR group.

no statistically significant differences between groups, except hepatic profiles such as ALP and ALT. Therefore, this result suggested that GBR might potentially protect liver function.

\section{Discussion}

In this study, we highlighted the experimental models to establish the potential effect of GBR on cardioprotection that is necessary to improve the therapeutic approaches following cardiovascular diseases. We constantly presented evidence indicating the cardioprotection effects of GBR in ex vivo studies and animal models. Firstly, we attempted to clarify the cardiomyocyte by detecting the cMYBPC 3 gene by PCR method (Figure 2). cMYBPC3 is a cardiac isoform of myosin binding protein $\mathrm{C}$, a sarcomeric protein, and plays a crucial role in regulating cardiac contraction. ${ }^{31}$ It has been reported that cMYBPC 3 mutation was associated not only in familial hypertrophic cardiomyopathy but also found to be dephosphorylated in I/R injury. ${ }^{30}$ The experiment of primary porcine cardiomyocytes established a significant increase of cell viability in cotreatment of GBR and cardioplegic solution
(Figure 5). Interestingly, we found that adding GBR into cardioplegia solution effectively preserved myocardial function similar with standard cardioplegic solution during openheart surgery under CBP in the porcine experimental model (Figure 5). Furthermore, animals that received GBR could reduce the parameters of cardiopulmonary bypass-related myocardial ischemic-reperfusion injury (Tables 1-3). The data obtained from this study provided a novel insight into the cardioprotective activity of GBR due to its biologically active compounds. The in vivo model established by openheart surgery can mimic the pathophysiological changes during ischemic-reperfusion. However, the in vivo model has a disadvantage in the number of laboratory animals. Although the animal's number should increase for analyzing our data, we still gain valuable results in understanding the cardioprotection induced by germinated brown rice treatment.

Bio-functional foods, especially GBR, have gained extensive attention due to their health-enhancing properties. ${ }^{13,15-23}$ However, a few previous studies prosperously published the potential effects of GBR on cardiac protection, including anti-inflammation, anti-oxidant, and anti-arrhythmia. ${ }^{21-23}$ They found that GBR demonstrated the cardio-protective effects against ischemic- reperfusion injury both in vitro and in vivo experiment from chronic myocardial injury. A study in $\mathrm{H} 9 \mathrm{c} 2$ cells revealed that GBR exerted its cardioprotective effects by preserving mitochondria function regarding mitochondria membrane potential and mitochondria respiration. ${ }^{23}$ The fact that possible mechanism by which p-38 MAPK pathway plays a critical role in cardioprotection of GBR against apoptosis. ${ }^{22}$ Also, as previous study demonstrated the bioactive compounds of GBR protect cardiac cells against $\mathrm{I} / \mathrm{R}$ injury via reducing the necrotic cell death and
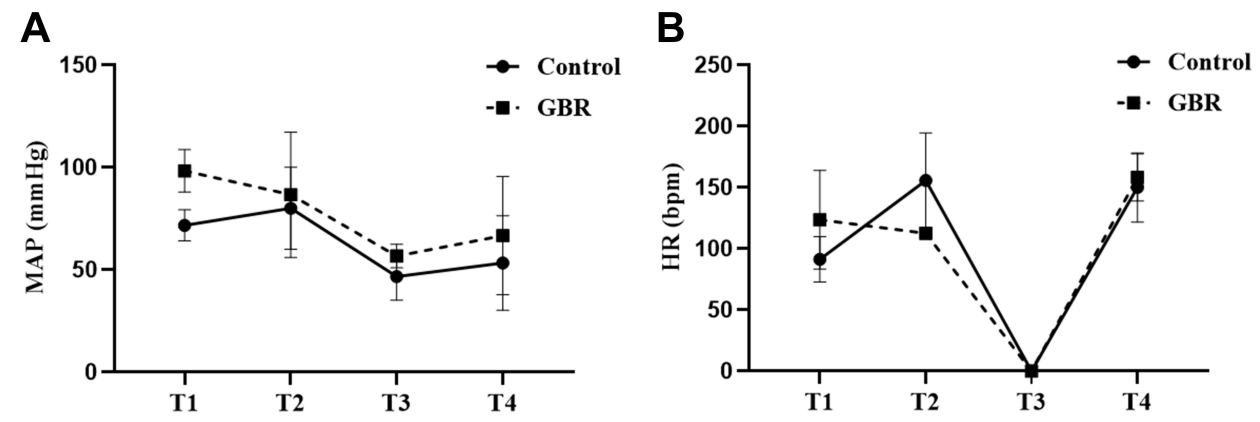

Figure 6 Effect of GBR treatment on hemodynamic data of the animal model. (A) Mean arterial pressure (mmHg), (B) Heart rate (bpm). Each group's data collection was divided into four times; $\mathrm{TI}=$ before $\mathrm{CPB}, \mathrm{T} 2=$ during $\mathrm{CPB}, \mathrm{T} 3=$ aortic clamp-on, $\mathrm{T} 4=$ aortic clamp off. The dark circle line represented the data of the control group $(\mathrm{N}=3)$. The square dark line represented the data of the GBR group $(\mathrm{N}=3)$. All data expressed the mean $\pm S D$. Two-way ANOVA analyzed data. $p<0.05$ was considered a statistically significant difference.

Abbreviations: MAP, mean arterial pressure; $\mathrm{HR}$, heart rate; $\mathrm{mmHg}$, millimeters of mercury; bpm, beat per minute; CPB, cardiopulmonary bypass. 
Table 2 Arterial Blood Gas Characteristics of Animals

\begin{tabular}{|c|c|c|c|c|}
\hline Parameters & TI & T2 & T3 & T4 \\
\hline $\mathrm{pH}$ & $7.4 \pm 0.02$ & $7.5 \pm 0.04$ & $7.5 \pm 0.06$ & $7.4 \pm 0.03$ \\
\hline \multicolumn{5}{|l|}{ CON } \\
\hline GBR & $7.3 \pm 0.01$ & $7.6 \pm 0.08$ & $7.5 \pm 0.09$ & $7.4 \pm 0.06$ \\
\hline $\mathrm{P}_{\mathrm{CO} 2}(\mathrm{mmHg}) \mathrm{CON}$ & $59.2 \pm 3.8$ & $43.7 \pm 3.5$ & $43.2 \pm 5.9$ & $46.3 \pm 5.5$ \\
\hline GBR & $50.6 \pm 13.9$ & $38.0 \pm 9.9$ & $37.2 \pm 11.05$ & $40.1 \pm 10.3$ \\
\hline $\mathrm{P}_{\mathrm{O} 2}(\mathrm{mmHg}) \mathrm{CON}$ & $435.6 \pm 44.05$ & $509.9 \pm 60.6$ & $509.6 \pm 12.7$ & $504.2 \pm 41.4$ \\
\hline GBR & $368.5 \pm 54.3$ & $573.6 \pm \mid 4.6$ & $560.6 \pm 10.4$ & $550.6 \pm 6.7$ \\
\hline $\mathrm{SpO}_{2}(\%) \mathrm{CON}$ & $99.7 \pm 0.03$ & $99.65 \pm 0.04$ & $99.3 \pm 0.2$ & $99.6 \pm 0.1$ \\
\hline GBR & $99.6 \pm 0.03$ & $99.3 \pm 0.05$ & $99.2 \pm 0.1$ & $99.2 \pm 0.3$ \\
\hline $\mathrm{Na}^{+}(\mathrm{mmol} / \mathrm{L}) \mathrm{CON}$ & $144.3 \pm 1.3$ & $138.4 \pm 1.8$ & $126.2 \pm 7.2$ & $133.6 \pm 1.8$ \\
\hline GBR & $132.2 \pm 3.6$ & $139.6 \pm 2.5$ & $134.5 \pm 3.4$ & $137.5 \pm 0.8$ \\
\hline $\mathrm{K}^{+}(\mathrm{mmol} / \mathrm{L}) \mathrm{CON}$ & $3.3 \pm 0.01$ & $4.3 \pm 0.03$ & $5.1 \pm 0.4$ & $5.3 \pm 0.6$ \\
\hline GBR & $3.3 \pm 0.1$ & $4.2 \pm 0.5$ & $4.4 \pm 0.3$ & $4.6 \pm 0.2$ \\
\hline Lactase (mmol/L) CON & $5.5 \pm 0.8$ & $10.6 \pm 0.3$ & $|2.4 \pm 4|$. & $14.4 \pm 2.8$ \\
\hline GBR & $9.2 \pm 0.8$ & $8.2 \pm 1.7$ & $9.1 \pm 1.4$ & $9.2 \pm 1.5$ \\
\hline
\end{tabular}

Abbreviations: CON, control group; GBR, Adding GBR group.

Table 3 Blood Chemistry Profiles of Animals

\begin{tabular}{|l|c|c|c|c|}
\hline Parameters & CON & GBR & p-value & Reference Range \\
\hline WBC $\left(\times 10^{3} / \mu \mathrm{l}\right)$ & $15.04 \pm 0.66$ & $17.92 \pm 1.62$ & 0.28 & $6-17$ \\
Platelets (gm\%) & $497.66 \pm 37.13$ & $533.50 \pm 53.42$ & 0.65 & $2-9 \times 10^{5}$ \\
Hematocrit (\%) & $29.66 \pm 0.88$ & $25.00 \pm 3.03$ & 0.16 & $30-35$ \\
ALP (U/L) & $201.33 \pm 3.76$ & $138.75 \pm 29.89$ & $0.03^{*}$ & $<200$ \\
ALT (U/L) & $60.66 \pm 23.7$ & $16.00 \pm 3.03$ & $0.03^{*}$ & $<89$ \\
BUN (mg\%) & $10.23 \pm 2.58$ & $13.88 \pm 1.82$ & 0.66 & $<34.5$ \\
Creatinine (mg\%) & $1.13 \pm 0.06$ & $1.11 \pm 0.13$ & 0.35 & $<1.8$ \\
Plasma protein $(/ \mu \mathrm{L})$ & $6.33 \pm 0.33$ & $6.1 \pm 0.31$ & 0.94 & $5-7.5$ \\
\hline
\end{tabular}

Abbreviations: CON, control group; GBR, Adding GBR group; WBC, White blood cell; ALP, Alkaline phosphatase; ALT, Alanine aminotransferase; BUN, Blood urea nitrogen.

inflammatory cytokines such as $\mathrm{LDH}$ and IL-4. ${ }^{17}$ Consistent with a previous study, GBR is able to decrease and obviate the reactive oxygen species (ROS) which is excessively produced during cardiac reperfusion injury and related to the inflammation process. ${ }^{22}$ Accumulated with the present in vivo study, we represented that GBR treatment prompted cell viability in isolated primary porcine cardiomyocytes against sI/R injury. Therefore, it provides the ability on anti-inflammatory action.

Importantly, there were several bioactive compounds of GBR that assisted the health-advantage activities. ${ }^{15,22}$ The significant active substrates of GBR extract have been demonstrated as anti-inflammatory, anti-arrhythmia, antidiabetic properties, anti-obesity, and anti-oxidant. ${ }^{13-21,32}$ It seems that the anti-inflammatory effect of GBR is due to enrichment of flavonoid and phenolic compound contents. It has been documented that flavonoid significantly prohibited a number of inflammatory mediators and prevents the synthesis of prostaglandins. ${ }^{33}$ The compound which contributes to these effects of GBR is not yet fully clarified. In addition, the present study indicated a maintenance of liver function by declining of ALP and ALT on animals receiving GBR. It has been shown that high flavonoid and phenolic compound in ginkgo biloba extracts exhibited an elimination of lipid synthesis and liver secretion in non-alcoholic fatty liver disease via ameliorating endoplasmic reticulum stress signals activation. $^{34}$ Indeed, clinical parameters of hepatic profiles characterized the damage in liver such as hepatocyte 
inflammation, oxidative stress, and liver injury. ${ }^{35}$ Therefore, we can assume that GBR exhibits an anti-inflammatory activity via preserving liver function.

According to the present findings in primary porcine cardiomyocytes, GBR co-treatment with cardioplegic solution provided cardioprotection similar to a cardioplegic solution alone. Hence, we further aimed to investigate the protective effect of GBR on cardiac surgery under CPB. Cardiac surgery under $\mathrm{CPB}$ is the most common method that significantly applies in clinical practice. CPB is non-physiologic circulation, leading to adverse effects such as organ dysfunction. ${ }^{36}$ Due to that, it has also been reported that open-heart surgery under CPB eventually triggered an acute systemic inflammation response. ${ }^{37}$ Myocardial damage during the surgical procedure is one of the main contributors to morbidity and mortality after heart surgery. ${ }^{38}$ Therefore, myocardial protection is strictly concerned during the operation. Literally, there are two major principles for myocardial protection, including cardioplegic solution and the hypothermia process. ${ }^{8}$ Both principles result in the reduction of cellular metabolism and energy consumption, and myocardial preservation. ${ }^{39}$ A variety of cardioplegic types have been applied depending on the hospital center.

In this animal study model, we preferred the experiment in the porcine open-heart surgery. St. Thomas cardioplegic solution $(20 \mathrm{cc} / \mathrm{kg})$, a standard cardioplegic solution, was administered for 20 minutes with mild hypothermia $\left(32^{\circ} \mathrm{C}\right)$ to arrest the heart and preserve the myocardium. At the same time, animals in the cotreatment group received the cardioplegic solution mixed with GBR $(1 \mathrm{mg} / \mathrm{kg})$ as the same protocol. The clinical parameters related to a surgical procedure on the effect of GBR cotreatment with a cardioplegic solution were reported. Our results demonstrated that animals in the GBR group exhibited a more significant characteristic of cardiopulmonary bypass surgery parameters such as cooling time, rewarming time, and $\mathrm{CPB}$ time (Table 1). In agreement with a previous study, they reported that less CPB time with hypothermia significantly improved organ perfusion and reduced the inflammatory response. ${ }^{40}$ Together with a study in ischemic stroke mice, the researcher documented a therapeutic hypothermia owing to its antiinflammatory activity. ${ }^{41}$ Regarding the present study, we performed open-heart surgery under CPB with hypothermia to preserve myocardial function. Therefore, the application of GBR with standard cardioplegic solution potentially showed a similar pattern on myocardial protection when compared to animals giving only standard cardioplegic solution. It seems like that GBR provided a protective effect against myocardial damage during open-heart surgery under CPB due to its bioactive compounds and acted as an anti-inflammatory response.

Aside from that, GBR combined with cardioplegic solution significantly exhibited a significant reduction of hepatic profiles, ALT and ALP with shorter CPB time, cooling time, and rewarming time (Table 3). Indeed, the pathogenesis of liver dysfunction following open-heart surgery depends on multi-factors. ${ }^{36,42}$ A previous study has reported the association of prolonged CPB time and the increase of liver enzymes. ${ }^{39}$ As mentioned above, GBR might demonstrate a liver preservation caused by an anti-inflammatory activity.

To collaborate with the result in this study, this finding demonstrated an excellent clinical parameters trend with arterial blood gas characteristics (Table 2) and the percentage of hematocrit, creatinine, and plasma protein (Table 3) in adding the GBR group. Several studies explained the systemic inflammatory response and oxidative stress from surgery under CPB (36). Lactate production is an end-step of anaerobic metabolism that can refer to adequate myocardial protection and sufficient organ perfusion by $\mathrm{CPB}$ during open-heart surgery. ${ }^{43}$ Consistent with our finding, animals in GBR groups gently exhibited a lower lactate production level throughout the surgical procedure. This data suggested that GBR might enhance organ perfusion and get rid of lactic acidosis undergoing open-heart surgery.

Similarly, mean arterial pressure and heart rate were well improved in animals receiving GBR but not a statistically significant difference (Figure 6). This result is consistent with the previous finding that consumption of GBR was capable of heart function as anti-arrhythmia activity in the chronic myocardial ischemia model. ${ }^{21}$ These findings led to the idea that GBR cotreatment with the cardioplegic solution could be more significant for cardioprotection during cardiac surgery under $\mathrm{CPB}$.

\section{Conclusion}

This study demonstrates the protective effects of GBR in primary cell culture against simulated ischemic-reperfusion injury. The cardio-protection of GBR was also investigated by open heart surgery under CPB. This study concluded that GBR cotreatment with standard cardioplegic solution might have exhibited a similar efficacy on myocardial protection during the surgical operation. However, definitive proof of cardiac protection of GBR is required further investigation. 


\section{Ethics Approval}

The study was approved by the Ethical Committee, Kasetsart University, Thailand (ACKU-62-VET-059).

\section{Acknowledgments}

The authors are grateful to Kasetsart University Veterinary Teaching Hospital, Faculty of Veterinary Medicine, Kasetsart University, for providing facilities for the study. We acknowledge Terumo corporation and Technomed USA Inc. for supporting surgical equipment.

\section{Funding}

This study was supported by National Research Council of Thailand for The Royal Golden Jubilee Ph.D. Program (grant no. PHD/0142/2561).

\section{Disclosure}

Dr Soontaree Petchdee reports a patent pending: patent number; 1803002749 The authors reported no conflicts of interest for this work.

\section{References}

1. Mensah GA, Roth GA, Fuster V. The Global Burden of Cardiovascular Diseases and Risk Factors. $\mathrm{J}$ Am Coll Cardiol. 2019;74(20):2529-2532. doi:10.1016/j.jacc.2019.10.009

2. Kaptoge S, Pennells L, De Bacquer D; Group WCRCW. World Health Organization cardiovascular disease risk charts: revised models to estimate risk in 21 global regions. Lancet Glob Health. 2019;7 (10):e1332-e45. doi:10.1016/S2214-109X(19)30318-3

3. Chei CL, Yamagishi K, Tanigawa T, et al. Metabolic Syndrome and the Risk of Ischemic Heart Disease and Stroke among Middle-Aged Japanese. Hypertension Res. 2008;31(10):1887-1894. doi:10.1291/ hypres. 31.1887

4. Fakhrzadeh H, Sharifi F, Alizadeh M, et al. Relationship between insulin resistance and subclinical atherosclerosis in individuals with and without type 2 diabetes mellitus. J Diabetes Metab Disord. 2015;15:41. doi:10.1186/s40200-016-0263-5

5. Bowdish ME, D'Agostino RS, Thourani VH, et al. The Society of Thoracic Surgeons Adult Cardiac Surgery Database: 2020 Update on Outcomes and Research. Ann Thorac Surg. 2020;109(6):1646-1655. doi:10.1016/j.athoracsur.2020.03.003

6. Passaroni AC, Silva MA, Yoshida WB. Cardiopulmonary bypass: development of John Gibbon's heart-lung machine. Revista Brasileira De Cirurgia Cardiovascular. 2015;30(2):235-245. doi:10. 5935/1678-9741.20150021

7. Hessel EA. What's New in Cardiopulmonary Bypass. J Cardiothorac Vasc Anesth. 2019;33(8):2296-2326. doi:10.1053/j.jvca.2019.01.039

8. Abdouni AA. Myocardial Protection in Cardiac Surgery - What is the Ideal Method? Arq Bras Cardiol. 2020;115(2):251-252. doi:10.36 660/abc. 20200622

9. von Oppell UO, Du Toit EF, King LM, et al. St. Thomas' Hospital cardioplegic solution. Beneficial effect of glucose and multidose reinfusions of cardioplegic solution. J Thorac Cardiovasc Surg. 1991;102(3):405-412. doi:10.1016/S0022-5223(19)36523-7

10. Donnelly AJ, Djuric M. Cardioplegia solutions. Am J Hosp Pharm. 1991;48(11):2444-2460.
11. Bhakri KP, Mulholland J, Punjabi PP. Understanding innovations in the evolving practice of blood and crystalloid cardioplegia. Perfusion. 2014;29(6):505-510. doi:10.1177/0267659114524977

12. Carvajal C, Goyal A, Tadi P. Cardioplegia. StatPearls. Treasure Island (FL): StatPearls Publishing Copyright (C) 2020, StatPearls Publishing LLC; 2020.

13. Ravichanthiran K, Ma ZF, Zhang H, et al. Phytochemical Profile of Brown Rice and Its Nutrigenomic Implications. Antioxidants. 2018;7 (6). doi:10.3390/antiox7060071

14. Imam MU, Azmi NH, Bhanger MI, Ismail N, Ismail M. Antidiabetic properties of germinated brown rice: a systematic review. Evid Based Complement Alternat Med. 2012;2012:816501. doi:10.1155/2012/ 816501

15. Wu F, Yang N, Toure A, Jin Z, Xu X. Germinated brown rice and its role in human health. Crit Rev Food Sci Nutr. 2013;53(5):451-463. doi:10.1080/10408398.2010.542259

16. Lim SM, Goh YM, Mohtarrudin N, Loh SP. Germinated brown rice ameliorates obesity in high-fat diet induced obese rats. $B M C$ Complement Altern Med. 2016;16:140. doi:10.1186/s12906-0161116-y

17. Lim SM, Goh YM, Kuan WB, Loh SP. Effect of germinated brown rice extracts on pancreatic lipase, adipogenesis and lipolysis in 3T3-L1 adipocytes. Lipids Health Dis. 2014;13:169. doi:10.1186/ 1476-511X-13-169

18. Usuki S, Ito Y, Morikawa K, et al. Effect of pre-germinated brown rice intake on diabetic neuropathy in streptozotocin-induced diabetic rats. Nutr Metab. 2007;4:25. doi:10.1186/1743-7075-4-25

19. Shen KP, Hao CL, Yen HW, et al. Pre-germinated brown rice prevented high fat diet induced hyperlipidemia through ameliorating lipid synthesis and metabolism in C57BL/6J mice. J Clin Biochem Nutr. 2016;59(1):39-44. doi:10.3164/jcbn.15-117

20. Ho JN, Son ME, Lim WC, Lim ST, Cho HY. Anti-obesity effects of germinated brown rice extract through down-regulation of lipogenic genes in high fat diet-induced obese mice. Biosci Biotechnol Biochem. 2012;76(6):1068-1074. doi:10.1271/bbb.110666

21. Petchdee S, Laosripaiboon W, Jarussophon N. Cardiac protection of germinated brown rice extract in rabbit model of chronic myocardial infarction. Transl Anim Sci. 2020;4(2):txaa067. doi:10.1093/tas/ txaa067

22. Petchdee S, Laosripaiboon W, Jarussophon N, Kumphune S. CardioProtective Effects of Germinated Brown Rice Extract Against Myocardial Ischemia Reperfusion Injury. High Blood Press Cardiovasc Prev. 2020;27(3):251-258. doi:10.1007/s40292-020-00378-x

23. Demeekul K, Suthammarak W, Petchdee S. Bioactive Compounds from Germinated Brown Rice Protect Cardiomyocytes Against Simulated Ischemic/Reperfusion Injury by Ameliorating Mitochondrial Dysfunction. Drug Des Devel Ther. 2021;15:1055-1066. doi:10.2147/ DDDT.S294779

24. Parameswaran S, Kumar S, Verma RS, Sharma RK. Cardiomyocyte culture - an update on the in vitro cardiovascular model and future challenges. Can J Physiol Pharmacol. 2013;91(12):985-998. doi:10. 1139/cjpp-2013-0161

25. Tateishi K, Ashihara E, Honsho S, et al. Human cardiac stem cells exhibit mesenchymal features and are maintained through Akt/ GSK-3beta signaling. Biochem Biophys Res Commun. 2007;352 (3):635-641. doi:10.1016/j.bbrc.2006.11.096

26. Prompunt E, Sanit J, Barrere-Lemaire S, et al. The cardioprotective effects of secretory leukocyte protease inhibitor against myocardial ischemia/reperfusion injury. Exp Ther Med. 2018;15(6):5231-5242. doi:10.3892/etm.2018.6097

27. Godiksen MT, Granstrom S, Koch J, Christiansen M. Hypertrophic cardiomyopathy in young Maine Coon cats caused by the p.A31P cMyBP-C mutation--the clinical significance of having the mutation. Acta Vet Scand. 2011;53:7. doi:10.1186/1751-0147-53-7

28. Uechi M. Mitral valve repair in dogs. J Vet Cardiol. 2012;14 (1):185-192. doi:10.1016/j.jvc.2012.01.004 
29. Uechi M, Mizukoshi T, Mizuno T, et al. Mitral valve repair under cardiopulmonary bypass in small-breed dogs: 48 cases (2006-2009). J Am Vet Med Assoc. 2012;240(10):1194-1201. doi:10.2460/javma.240.10.1194

30. Flashman E, Redwood C, Moolman-Smook J, Watkins H. Cardiac Myosin Binding Protein C. Circ Res. 2004;94(10):1279-1289. doi:10.1161/01.RES.0000127175.21818.C2

31. Oakley CE, Chamoun J, Brown LJ, Hambly BD. Myosin binding protein-C: enigmatic regulator of cardiac contraction. Int J Biochem Cell Biol. 2007;39(12):2161-2166. doi:10.1016/j.biocel.2006.12.008

32. Amraei M, Ghorbani A, Seifinejad Y, Mousavi SF, Mohamadpour M, Shirzadpour E. The effect of hydroalcoholic extract of Teucrium polium L. on the inflammatory markers and lipid profile in hypercholesterolemic rats. J Inflamm Res. 2018;11:265-272. doi:10.2147/JIR.S165172

33. Mengie T, Mequanente S, Nigussie D, Legesse B, Makonnen E. Investigation of Wound Healing and Anti-Inflammatory Activities of Solvent Fractions of $80 \%$ Methanol Leaf Extract of Achyranthes aspera L. (Amaranthaceae) in Rats. $J$ Inflamm Res. 2021;14:1775-1787. doi:10.2147/JIR.S298244

34. Li LYL, Yang F, Zhao XL, Xue SJ, Gong FH. Ginkgo biloba Extract 50 (GBE50) Ameliorates Insulin Resistance, Hepatic Steatosis and Liver Injury in High Fat Diet-Fed Mice. $J$ Inflamm Res. 2021;14:1959-1971. doi:10.2147/JIR.S302934

35. Bessone F, Razori MV, Roma MG. Molecular pathways of nonalcoholic fatty liver disease development and progression. Cell Mol Life Sci. 2019;76(1):99-128. doi:10.1007/s00018-018-2947-0

36. Paparella D, Yau TM, Young E. Cardiopulmonary bypass induced inflammation: pathophysiology and treatment. An update. Eur J Cardiothorac Surg. 2002;21(2):232-244. doi:10.1016/s1010-7940(01)01099-5
37. Vejlstrup A, Moller AM, Nielsen CH, Damgaard D. Release of active peptidylarginine deiminase into the circulation during acute inflammation induced by coronary artery bypass surgery. J Inflamm Res. 2019;12:137-144. doi:10.2147/JIR.S198611

38. Yamamoto H, Yamamoto F. Myocardial protection in cardiac surgery: a historical review from the beginning to the current topics. Gen Thorac Cardiovasc Surg. 2013;61(9):485-496. doi:10.1007/s11748013-0279-4

39. Elassal AA, Al-Ebrahim K, Al-Radi O, et al. Myocardial Protection by Blood-Based Del Nido versus St. Thomas Cardioplegia in Cardiac Surgery for Adults and Children. Heart Surg Forum. 2020;23(5): E689-E95. doi:10.1532/hsf.3099

40. Sabzi F, Faraji R. Liver Function Tests Following Open Cardiac Surgery. J Cardiovasc Thorac Res. 2015;7(2):49-54. doi:10.15171/ jevtr.2015.11

41. Yu X, Feng Y, Liu R, Chen Q. Hypothermia Protects Mice Against Ischemic Stroke by Modulating Macrophage Polarization Through Upregulation of Interferon Regulatory Factor-4. J Inflamm Res. 2021;14:1271-1281. doi:10.2147/JIR.S303053

42. Chacon MM, Schulte TE. Liver Dysfunction in Cardiac Surgery What Causes It and Is There Anything We Can Do? J Cardiothorac Vasc Anesth. 2018;32(4):1719-1721. doi:10.1053/ j.jvca.2018.02.037

43. Minton J, Sidebotham DA. Hyperlactatemia and Cardiac Surgery. J Extra Corpor Technol. 2017;49(1):7-15.
Journal of Inflammation Research

\section{Publish your work in this journal}

The Journal of Inflammation Research is an international, peerreviewed open-access journal that welcomes laboratory and clinical findings on the molecular basis, cell biology and pharmacology of inflammation including original research, reviews, symposium reports, hypothesis formation and commentaries on: acute/chronic inflammation; mediators of inflammation; cellular processes; molecular mechanisms; pharmacology and novel anti-inflammatory drugs; clinical conditions involving inflammation. The manuscript management system is completely online and includes a very quick and fair peerreview system. Visit http://www.dovepress.com/testimonials.php to read real quotes from published authors.

Submit your manuscript here: https://www.dovepress.com/journal-of-inflammation-research-journal 